\title{
Ricerca e aggiornamento infermieristico in Nefrologia e Dialisi mediante internet
}

\author{
F. Armani, S. Bacciardi, I. Rossi \\ U.O. Nefrologia dei Trapianti e Dialisi 1, A.O.U. Pisana
}

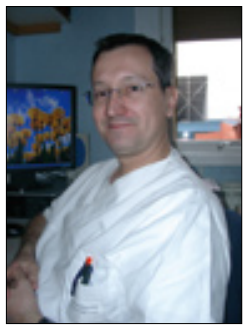

Fernando Armani

\section{Introduzione}

L'aggiornamento infermieristico si deve ritenere indispensabile dato che le conoscenze scientifiche e le innovazioni tecnologiche sono in costante $\mathrm{e}$ sempre più veloce evoluzione.

I modi per aggiornare le proprie conoscenze possono essere vari, come congressi, corsi, $\mathrm{FAD}$, testi e riviste scientifiche, e internet.

Internet è un sistema dal quale si possono attingere moltissime informazioni e ha molti vantaggi rispetto ad altri sistemi tradizionali:

- La rapidità delle risposte a quesiti.

- Lattualità, in quanto i siti sono sempre aggiornabili.

- L'economicità, perché i costi sono oramai minimi.

- Lubiquitarietà, perché consultabile da casa, dal lavoro, in viaggio e a qualsiasi ora del giorno e della notte.

Il principale limite rimane l'affidabilità della fonte di informazione che deve sempre essere verificata.

Sulla base di queste considerazioni ci siamo posti la domanda: «Gli infermieri usano internet per aggiornare le loro conoscenze, e se sì, sanno come utilizzarlo?».

Lo scopo del lavoro è stato quindi di rispondere al quesito indagando l'attitudine all'utilizzo dei siti di interesse nefrologico da parte degli infermieri di area nefrologica.

\section{Materiali e Metodi}

Lo studio ha incluso 90 infermieri di area nefrologica, operanti in corsia, ambulatorio e dialisi degli ospedali di Pisa, Livorno, Lucca, Lido di Camaiore (Versilia), Volterra, S. Miniato.
Gli infermieri sono stati reclutati in modo randomizzato dalla popolazione infermieristica descritta in Tabella I. Il questionario originale era composto da dieci domande a risposta multipla: Sì - No - In Parte, preparato per valutare le conoscenze del personale infermieristico sui siti internet di interesse nefrologico (Tab. II). A tutti i partecipanti è stato garantito l'anonimato.

Sono stati consegnati 15 test per ogni Centro, insieme con le informazioni per spiegare lo scopo dellindagine. La fase di stesura del test, la sua distribuzione e il ritiro è durata circa 45 giorni. Al termine del ritiro dei test è iniziata la fase di elaborazione dalla quale sono state generate le statistiche delle risposte e le elaborazioni e interpretazioni dei dati.

\section{Risultati}

Dai dati presenti nella Tabella II e nella Figura 1, è evidente come le risposte fornite dal campione indichino in modo chiaro che esiste una prevalenza di risposte "No" alle domande sull'uso di internet.

Se analizziamo più attentamente la Tabella II notiamo poi che le uniche domande che hanno ricevuto le risposte "Si" in percentuale maggiore rispetto al "No" sono state la 1 la 5 e la 6 , mentre quelle che hanno ricevuto un netto "No" alla risposta sono state la 2, 3, 7, 8, 9, $10 \mathrm{e}$ sono relativi a siti o servizi on-line in lingua inglese. La Tabella II riporta le singole risposte ai singoli quesiti.

\section{Discussione}

I risultati dell'indagine dimostrano una scarsa propensione degli intervistati all'uso di internet quale fonte di aggiornamento professionale. 
TABELLA I - CARATTERISTICHE DEL CAMPIONE UTILIZZATO PER LO STUDIO

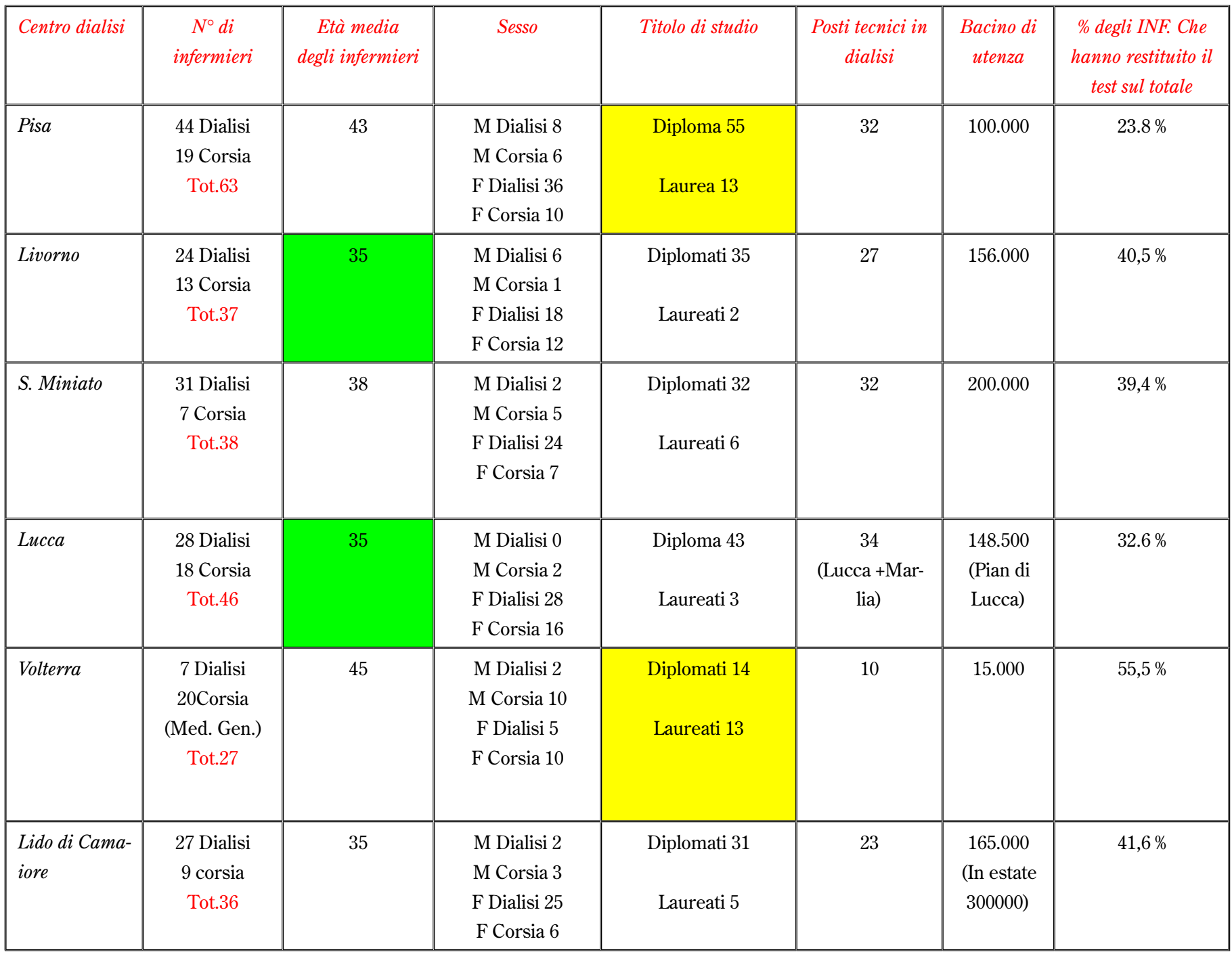

Il perché di una così scarsa confidenza del personale infermieristico all'uso di internet non è di facile interpretazione. Forse il primo punto che causa una distanza dalla rete è culturale, poi la probabile scarsa confidenza con la lingua inglese porta inevitabilmente a evitare i siti esteri.

L'età anagrafica del personale può avere importanza dato che i giovani hanno maggiore affinità nell'uso di internet; questo è emerso dall'analisi comparativa fra l'età media del personale dei Centri contattati e le risposte affermative date.

Altro elemento che è scaturito dall'analisi dei dati è che il titolo di studio di grado superiore, che nel caso del nostro campione corrispondeva alla laurea, non è elemento a favore della conoscenza di internet.
Ultimo elemento può essere la scarsa presenza di PC allinterno dei luoghi di cura a disposizione del personale infermieristico, che potrebbe favorire l'aggiornamento nei momenti in cui si riduce la mole di lavoro, senza ovviamente che venga meno la vigilanza verso il paziente.

Internet è un sistema veloce e preciso per individuare materiale scientifico da utilizzare per la produzione di lavori scientifici, ma è importante che le fonti siano attendibili, pertanto è fondamentale reperire dati da siti riconosciuti come affidabili. Preferibilmente il sito dovrebbe avere un comitato scientifico che vagli le informazioni contenute nel sito stesso e che ne sia garante.

Per facilitare un approccio a internet abbiamo selezionato dei siti in base alla loro importanza e affidabilità. 


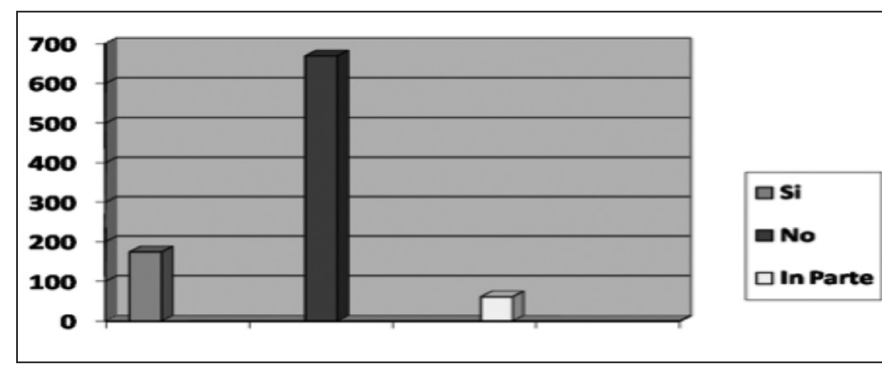

Fig. 1

TABELLA II - RISULTATI DELLE RISPOSTE DATE DAL CAMPIONE

\begin{tabular}{lccc}
\hline & SI & NO & In Parte \\
\hline 1) Ritieni di conoscere in modo sufficiente l'uso di internet? & $35 \%$ & $28 \%$ & $35 \%$ \\
2) Hai mai usato il servizio My NCBI di PUB MED? & $3,90 \%$ & $95 \%$ & $1 \%$ \\
3) Hai mai usato il servizio MESH data base di PUB MED? & $3,90 \%$ & $95 \%$ & $1 \%$ \\
4) Hai mai attinto informazioni dal sito GLOBALDIALYSIS? & $10,00 \%$ & $86,60 \%$ & $3,30 \%$ \\
5) Sai della possibilità di scaricare da internet gratuitamente & $53 \%$ & $41 \%$ & $5,10 \%$ \\
$\quad$ un testo scientifico in lingua italiana sulla dialisi? & & & \\
6) Sai che all'interno del sito dell'I.P.A.S.V.I. esiste una & $65,90 \%$ & $28,50 \%$ & $0,80 \%$ \\
$\quad$ RASSEGNA STAMPA? & & $93 \%$ & $0,80 \%$ \\
7) Hai mai letto i periodici on-line dell'associazione A.N.N.A.? & $6,00 \%$ & $93 \%$ & $0,80 \%$ \\
(American Nephrology Nurses Association) & & & \\
8) Hai mai letto articoli del forum multidisciplinare RENAL PRO? & $6,00 \%$ & $93 \%$ \\
9) Conosci il sito dell'associazione A.A.K.P & $6,00 \%$ & $93 \%$ & $0,80 \%$ \\
$\quad$ (Amercan Association of Kidney Patient)? & $6,00 \%$ & $93 \%$ & $0,80 \%$ \\
10) Conosci il portale NEPHROWORLD.COM? & & \\
\hline
\end{tabular}

\begin{tabular}{|l|c|c|c|}
\hline PERCENTUALE DELLE RISPOSTE RICEVUTE & Sì & No & In Parte \\
\hline & $19,24 \%$ & $74 \%$ & $6,74 \%$ \\
\hline
\end{tabular}

\section{SELEZIONE DEI SITI}

\section{Cenni storici}

Internet nasce negli U.S.A. negli anni sessanta durante la guerra fredda allo scopo di consentire gli scambi di informazioni fra le varie basi militari americane anche in caso di attacco nucleare.

Nota per il lettore:

Internet è un sistema di divulgazione in continua evoluzione, quindi le informazioni contenute in questo articolo anche se aggiornate potrebbero aver subito variazioni.

Siti in lingua italiana

www.renalgate.it

Sito molto conosciuto in ambiente nefrologico adatto anche ai pazienti e non solo per il personale sanitario.

Si possono trovare informazioni su dialisi, accessi vascolari compresa la fistola $\mathrm{A} / \mathrm{V}$ e sulla dieta.

www.edtna-erca.it

Sito dell'Associazione Europea degli infermieri di dialisi e trapianti, Associazione Europea per il trattamento delle malattie renali, filiale italiana. In questo sito si possono avere informazioni sull'attività dell'associazione, cliccando sul link Journal renal care si possono leggere i titoli degli articoli pubblicati sulla rivista.

Nell'area "Documenti e pubblicazioni” si potranno leggere gli atti dei congressi dell'associazione con i relativi lavori. www.ipasvi.it

Sito generalista del collegio IPASVI. In questo sito suggeriamo di visionare la "Rassegna stampa" dove si possono leggere articoli pubblicati su molte testate giornalistiche di quotidiani e periodici. Questi articoli trattano gli argomenti più disparati ma hanno in comune la figura dell'infermiere, esempi:

Per medici e infermieri record di disturbi psicologici (da Adnkronos 16/2/2010).

Perché l'infermiere non è tanto amato (da Italia oggi 05/01/2009).

www.emodialisi.altervista.org

In questo sito ci si può documentare sulla storia della dialisi.

www.sin-italy.org

In questo sito della Società Italiana di Nefrologia trova ospitalità free-online il giornale di Tecniche Nefrologiche \& Dialitiche.

www.societaitalianatrapiantidiorgano.com

Sito dal quale prendere informazioni sui trapianti, per conoscerne gli aspetti legislativi, leggere la rivista Trapianti (solo se si è iscritti all'associazione) oppure conoscere gli indirizzi e numeri di telefono dei vari Centri trapianti d'Italia.

Siti in lingua inglese

Se non si avesse grande conoscenza della lingua inglese è consigliabile farsi aiutare dai traduttori automatici come quello di Google.

www.annanurse.org

Sito dell'associazione American Nephrology Nurses Association: Associazione di infermieri degli USA. Ottimo sito dal quale si possono attingere moltissime informazioni.

Cliccando sulla voce Nephrology Nursing Journal è possibile leggere gli articoli del loro periodico.

www.edtnaerca.org

Sito ufficiale dell'Associazione Europea Infermieri di area Nefrologica, Dialisi e Trapianti. Sito importante con molte pagine. Ponete la vstra attenzione su due servizi: uno è il Journal Renal Care (periodico scientifico dell'associazione) dal quale è possibile leggere gli abstract dell'ultimo numero pubblicato; l'altro servizio è quello con la possibilità di iscriversi al forum professionale RenalPro.

www.globaldialysis.com

Sito dedicato a quei pazienti con problemi di insuffi- 
cienza renale ma che comunque intendono viaggiare. Esiste la possibilità di conoscere gli indirizzi di molti Centri dialisi nel mondo o leggere i report scritti da altri pazienti dei loro viaggi. Cliccando su un apposito link è possibile acquistare abbigliamento specifico per chi fa dialisi.

Miscellanea di siti in lingua italiana e inglese

Fing

www.nursesdirect.com

Sito che vende articoli da regalo e per uso professionale come magliette, tazze, ciabatte, zoccoli ecc. che hanno in comune la figura dellinfermiere.

www.youtube.it

Sito molto famoso dal quale si possono visionare molti video scientifici sulla dialisi.

www.lightersideofdialysis.com

Questo è un sito di presentazione e vendita di un libro di vignette ironiche improntate sulla dialisi.

L' esperienza nel reperire materiale scientifico da internet è un elemento molto importante; ovviamente per poterne reperire bisogna prima avere la pazienza di imparare i sistemi di ricerca e memorizzare gli indirizzi dei siti che ci interessano maggiormente.

Ogni infermiere come un professionista dovrebbe non solo aggiornare le proprie conoscenze attraverso internet ma andare oltre e quindi consultare riviste, periodici, libri, frequentare corsi e congressi, non limitandosi alle conoscenze ricevute dagli studi di base o derivati dalla propria personale esperienza.

Per arrivare a questo livello di sensibilità verso la conoscenza occorre tempo e stimolo da parte degli infermieri verso gli infermieri. Questo non è un gioco di parole ma riteniamo che l'esempio silenzioso di alcuni infermieri che studiano e si aggiornano sia di stimolo verso gli altri che vedendo che non esiste solo la pratica clinica o le tecniche lavorative legate alla tradizione siano spronati ad adottare altre metodologie di lavoro e organizzazioni diverse e migliori. Questo è un punto importante perché le metodologie di lavoro troppo spesso sono legate alla tradizione e quando si cerca di inserire tecniche nuove derivate da studi che ne certificano l'affidabilità c'è spesso resistenza e la giustificazione classica che viene data da chi si oppone al cambiamento è:

«Si è sempre fatto cosi, perché cambiare?».

Questo atteggiamento è criticabile perché non per- mette alla professione di evolvere è può in alcuni casi portare a problemi anche di natura medico-legale.

Proponiamo di implementare dei corsi dedicati agli infermieri nei quali si insegnino le tecniche e i sistemi per individuare informazioni che poi serviranno sia nella ricerca infermieristica sia nella pratica clinica.

Altro elemento che potrebbe portare all'uso di internet è un maggior numero di computer collegati alla rete all'interno della Aziende Ospedaliere e Sanitarie locali.

Riteniamo che la semplice indagine multicentrica fatta in questo lavoro abbia messo in evidenza l'elemento più importante cioè la disponibilità dei colleghi a voler migliorare le proprie conoscenze e permettere a un altro collega di indagare sui punti deboli della propria cultura scientifica ed eventualmente mettere in evidenza le proprie lacune culturali.

Durante la distribuzione e la raccolta dei test abbiamo sempre trovato molta disponibilità e interesse in tutti $\mathrm{i}$ Centri da noi contattati. L'impressione è che l'interesse per l'aggiornamento sia presente ma sopito negli infermieri e che andrebbe solamente fatto emergere.

\section{Conclusioni}

I risultati dell'indagine dimostrano una scarsa propensione degli infermieri intervistati all'uso di internet quale fonte di aggiornamento professionale.

Durante la distribuzione e la raccolta dei test abbiamo comunque sempre trovato molta disponibilità e interesse in tutti i Centri da noi reclutati. L'impressione è che l'interesse per l'aggiornamento sia presente ma sopito negli infermieri e che andrebbe fortemente stimolato.

\section{Ringraziamenti}

Ringraziamo vivamente $\mathrm{i}$ colleghi che hanno risposto con precisione e puntualità alle domande del test multicentrico.

\section{Riassunto}

Lo scopo del lavoro è stato quello di verificare l'attitudine all'utilizzo dei siti di interesse nefrologico da parte degli infermieri di area nefrologica.

Lo studio ha incluso 90 infermieri dell'area nefrologica, operanti in corsia, ambulatorio e dialisi degli ospedali di: Pisa, Livorno, Lucca, Lido di Camaiore (Versilia), Volter- 
ra, S. Miniato.

Il questionario era composto da dieci domande a risposta multipla Sì - No - In Parte, preparato per valutare le conoscenze del personale infermieristico sui siti internet di interesse nefrologico.

I risultati dell'indagine dimostrano una scarsa propensione degli infermieri intervistati all'uso di internet quale fonte di aggiornamento professionale.

Durante la distribuzione e la raccolta dei test abbiamo sempre trovato molta disponibilità e interesse in tutti $\mathrm{i}$ Centri da noi reclutati. L’impressione è che l'interesse per l'aggiornamento sia presente ma sopito negli infermieri e che andrebbe solamente fatto emergere.

Parole chiave: Internet, Siti , Infermieri, Aggiornamento

\section{Sitografia}

www.renalgate.it www.edtna-erca.it www.ipasvi.it www.emodialisi.altervista.org www.sin-italy.org www.societàitalianatrapiantidiorgano.com www.google.it www.annanurse.org www.edtnaerca.org www.globaldialysis.com www.nursesdirect.com www.youtube.it www.lightersideofdialysis.com

Indirizzo degli Autori:

Fernando Armani, MD

Via Giorgio Vasari 8

56126 Pisa

ferarm2005@yahoo.it 